\title{
Depression, anxiety and their comorbidity in the Swedish general population: Point prevalence and the effect on health-related quality of life
}

Background: Depression and anxiety disorders are major world-wide problems. There are no or few epidemiological studies investigating the prevalence of depression, generalized anxiety disorder and anxiety disorders in general in the Swedish population.

Methods: Data were obtained by means of a postal survey administered to 3001 randomly selected adults. After two reminders response rate was $44.3 \%$. Measures of depression and general anxiety were the 9-item Patient Health Questionnaire Depression Scale (PHQ-9) and the 7-item Generalized Anxiety Disorder Scale (GAD-7). The PHQ-9 identified participants who had experienced clinically significant depression (PHQ-9 $\geq 10$ ), and who had a diagnosis of major depression (defined by using a PHQ-9 scoring algorithm). Clinically significant anxiety was defined as having a GAD-7 score $\geq 8$. To specifically measure generalized anxiety disorder, the Generalized Anxiety Disorder Questionnaire-IV (GAD-Q-IV) was used with an established cut-off. Health-related quality of life was measured using the EuroQol (EQ-5D). Experiences of treatments for psychiatric disorders were also assessed.

Results: Around 17.2\% (95\% CI: 15.1 - 19.4) of the participants were experiencing clinically significant depression (10.8\%; 95\% CI: 9.1 - 12.5) and clinically significant anxiety (14.7\%; 95\% CI: 12.7 - 16.6). Among participants with either clinically significant depression or anxiety, nearly $50 \%$ had comorbid disorders. The point prevalence of major depression was 5.2\% (95\% CI: 4.0 - 6.5), and 8.8\% (95\% CI: 7.3 - 10.4) had GAD. Among those with either of these disorders, 28.2\% had comorbid depression and GAD. There were in general significant gender differences, with more women having a disorder compared to men. Among those with depression or anxiety, only between half and two thirds had any treatment experience. Comorbidity was associated with higher symptom severity and lower health-related quality of life. 
Conclusions: Epidemiological data from the Swedish community collected in this study provide point prevalence rates of depression, anxiety disorders and their comorbidity. These conditions were shown in this study to be undertreated and associated with lower quality of life, that need further efforts regarding preventive and treatment interventions. 
1 AUTHOR LIST:

2 Robert Johansson ${ }^{1 \S}$, Per Carlbring ${ }^{2}$, Åsa Heedman ${ }^{1}$, Björn Paxling ${ }^{1}$, Gerhard

3 Andersson $^{1,3,4}$

4

$5 \quad{ }^{1}$ Department of Behavioural Sciences and Learning, Linköping University, Linköping,

6 Sweden

$7 \quad{ }^{2}$ Department of Psychology, Stockholm University, Stockholm, Sweden

$8{ }^{3}$ Department of Clinical Neuroscience, Psychiatry section, Karolinska Institutet,

9 Stockholm, Sweden

$10{ }^{4}$ Swedish Institute for Disability Research, Linköping University, Linköping, Sweden

11

$12{ }^{\S}$ Corresponding author

13

14 CORRESPONDING AUTHOR:

15 Robert Johansson

16 Department of Behavioural Sciences and Learning

17 Linköping University

18 SE-58183 Linköping

19 Sweden

20 Phone: +46 (0)13 282217

21 E-mail: robert.johansson@liu.se

22 


\section{Introduction}

2 Depression and anxiety disorders are major world-wide health problems that affects a

3 substantial amount of individuals every year (Ebmeier, Donaghey, \& Steele, 2006;

4 Kessler, Merikangas, \& Wang, 2007). In the US National Comorbidity Survey

5 Replication (NCS-R), lifetime prevalence of mood and anxiety disorders were $20.8 \%$

6 and 28.8\%, respectively (Kessler et al., 2007). Twelve-month prevalence estimates of

7 mood and anxiety range from $6.6 \%$ to $11.9 \%$ and $5.6 \%$ to $18.1 \%$ across surveys from

8 Europe, Australia and the US (Baumeister \& Härter, 2007). In Sweden in 1957, the

9 point prevalence of depression was estimated to be $4.7 \%$, based on data from the total

10 population ( $n=2612$ ) of Lundby, a small rural area in southern Sweden (Rorsman et

11 al., 1990). Using the national Swedish Twin Registry, lifetime prevalence for

12 depression was estimated to be $13.2 \%$ among men and $25.1 \%$ among women

13 (Kendler, Gatz, Gardner, \& Pedersen, 2006). In the Lundby study, lifetime prevalence

14 for depression was $27 \%$ among men and $45 \%$ among women, when participants were

15 followed from 1957 up to 1972 (Rorsman et al., 1990). Importantly, the Lundby study

16 did not use DSM criteria for major depression, which makes comparisons to

17 prevalence rates from other countries complicated (Rorsman et al., 1990). To our

18 knowledge, there exist no up-to-date point estimates of DSM-IV depression from the

19 Swedish general population.

21 For anxiety disorders, there is a 12-month prevalence study from the Swedish general

22 population regarding panic disorder (2.2\%; Carlbring, Gustafsson, Ekselius, \&

23 Andersson, 2002) and a point prevalence study on social phobia (15.6\%; Furmark et

24 al., 1999). In the NCS-R, the 12-month prevalence for these disorders were $2.7 \%$ and

25 6.8\%, respectively (Kessler, Chiu, Demler, Merikangas, \& Walters, 2005). From 
1 Sweden, there exist point prevalence data on generalized anxiety disorder (GAD)

2 from primary care (3.6\%; Allgulander \& Nilsson, 2003; Munk-Jørgensen et al., 2006).

3 This can be compared to NCS-R, where 12-month prevalence of GAD was 3.1\%

4 (Kessler et al., 2005). However, the point prevalence of GAD in the general Swedish

5 population seems unknown.

6

7 Comorbidity between mood and anxiety disorders is known to be common. For

8 example, among participants in the NCS-R that had a diagnosis of major depression,

$957.5 \%$ also met criteria for at least one anxiety disorder (Kessler et al., 2007). Other

10 epidemiological data suggests that $59.0 \%$ of individuals with GAD fulfill criteria for

11 major depression (Carter, Wittchen, Pfister, \& Kessler, 2001). This suggests that

12 comorbidity between depression and anxiety disorders is the rule rather than the

13 exception. Comorbidity has consistently been associated with a poorer prognosis and

14 greater demands for professional help (Albert, Rosso, Maina, \& Bogetto, 2008;

15 Schoevers, Deeg, van Tilburg, \& Beekman, 2005). In addition, comorbidity between

16 depression and anxiety seems strongly associated both with role impairment and

17 higher symptom severity (Kessler et al., 2003). Generally, psychiatric comorbidity is

18 known to affect various aspects of health-related quality of life (Carpentier et al.,

19 2009; Saarni et al., 2007; Sherbourne et al., 2010).

21 The aim of the present study was to investigate the point prevalence of depression,

22 GAD, anxiety disorders in general, and comorbidity in a representative Swedish

23 sample. We also investigated the effect of depression, anxiety and comorbidity on

24 health-related quality of life. 


\section{Materials \& Methods}

2 This study was carried out in accordance with the STROBE initiative for reporting

3 epidemiological data (Von Elm et al., 2007). Approval for the study was obtained

4 from the institutional review board at the Department of Behavioural Sciences and

5 Learning, Psychology section, Linköping University, Sweden. By responding to the

6 postal survey, participants gave their consent to take part in the study.

\section{Sample and data collection}

8 Data collection was done in the autumn of 2009. A total of 3001 participants aged

$9 \quad 18-70$ years were randomly selected from the Swedish population and address register

10 (SPAR). This register includes all persons who are registered as residents in Sweden,

11 both Swedish and non-Swedish citizens. Systematic sampling with a randomly chosen

12 starting point and a fixed interval was used as sampling technique. The researchers

13 conducting the study were not involved in the sampling.

15 A questionnaire (described below) was mailed by surface mail to each participant ( $n=$

16 3001), together with a stamped return envelope. There was also an explanatory letter,

17 in which the study was described. A web version of the survey was also constructed

18 and all participants could choose between the paper survey and the web survey.

19 Participants were also informed that anonymity was guaranteed and that all data

20 collected were to be used for research purposes only. About two weeks after initially

21 sending the letters, questionnaires and stamped return envelopes were sent out to

22 participants who had not yet responded $(n=2175)$. Thirty-nine participants could not

23 be reached by mail and their questionnaires were returned undelivered. In addition,

24 two questionnaires were returned because of insufficient knowledge of Swedish. A

25 total of 1329 (44.3\%) responded and were eligible for analysis. Forty-two of these 
1 questionnaires were returned using the Internet version of the survey. In cases where a

2 participant's response had missing values, all available data were used as long as a

3 complete score (e.g. the total score for an instrument) could be calculated. Details of

4 attrition can be found in Table 1-3. Among those who responded, mean age was 46.2

5 years $(S D=14.5)$ and 745 participants $(56.1 \%)$ were female. Fifty-two percent had

6 post-secondary education.

\section{Questionnaire design and measures of mental health}

8 The survey contained questions about demographics and treatment history. Moreover,

9 the survey contained established measures of depression, general anxiety, GAD and

10 health-related quality of life (HRQoL). These measures are described below. In total,

11 there were 28 questions in the survey. A mistake in the questionnaire design made the

12 question regarding educational level impossible to interpret whether answers were

13 given for ongoing or completed education.

\section{Depressive symptoms}

15 Symptoms of depression were measured using the 9-item Patient Health

16 Questionnaire Depression Scale (PHQ-9; Kroenke, Spitzer, \& Williams, 2001). The

17 PHQ-9 contains 9 items, with a total score ranging from 0 to 27, where each item is

18 scored 0 to 3 (0: Not at all; 1 : Several days; 2: More than half of the days; 3: Nearly

19 every day). Total scores of 0-4 indicate no depression, 5-9 mild depression, 10-14

20 moderate depression, 15-19 moderately severe depression and 20-27 severe

21 depression (Kroenke et al., 2001). Psychometric properties for the PHQ-9 have been

22 shown to be good, with an internal consistency in the range Cronbach's $\alpha=.86-.89$

23 and a test-retest reliability of $r=.84$ (Kroenke, Spitzer, Williams, \& Löwe, 2010). As

24 the nine items corresponds to the DSM-IV criteria for depression, the PHQ-9 can be

25 used with a diagnostic algorithm to ascertain a probable diagnosis of major 
1 depression. A diagnosis is made if five or more of the nine depressive symptoms are

2 reported to be present (defined as having an item score of $\geq 2$ ), and one of the

3 symptoms is anhedonia or depressed mood (having a score of $\geq 2$ on question 1 or 2 ).

4 Another way of detecting depression is to use a cut-off score. A PHQ-9 score of $\geq 10$

5 is an established cut-off and seem to be the optimal balance between sensitivity (88\%)

6 and specificity (88\%) when detecting depression (Kroenke et al., 2001). The more

7 strict PHQ-9 score of $\geq 15$ yields a higher specificity (95\%), but lower sensitivity

8 (68\%) (Kroenke et al., 2001).

10 The PHQ-9 has been shown to be a valid instrument when measuring current

11 depression in the general population (Kroenke et al., 2009). It has also been used to

12 provide prevalence estimates in population-based studies conducted in Germany

13 (Martin, Rief, Klaiberg, \& Braehler, 2006), Australia (Pirkis et al., 2009) and Canada

14 (Patten \& Schopflocher, 2009). All these studies used the diagnostic algorithm

15 mentioned above, and the study by Pirkis et al. (2009) did also describe prevalence

16 rates using the PHQ-9 cut-off score 10. In this study, we used the PHQ-9 diagnostic

17 algorithm to provide an estimate of current major depression in the general

18 population. We also used the cut-off score of $\geq 10$ to estimate the rate of 'clinically

19 significant depression' (Kroenke et al., 2001; Pirkis et al., 2009). The PHQ-9 was

20 chosen as a measure of depression over the established 21-item Beck Depression

21 Inventory-II (BDI-II; Beck, Steer, \& Brown, 1996) foremost because of length, but

22 also due to copyright issues. Strong associations have been found between the PHQ-9

23 and the BDI-II in various populations, indicating a convergent validity between the

24 instruments (Dum, Pickren, Sobell, \& Sobell, 2008; Martin et al., 2006; Titov et al., 25 2011). 


\section{Anxiety disorders}

3 The 7-item Generalized Anxiety Disorder Scale (GAD-7; Spitzer, Kroenke, Williams,

4 \& Löwe, 2006) was included as a questionnaire regarding anxiety disorders in

5 general. It is a 7-item measure, with items scored 0-3, and a total score of 21.

6 Originally developed as a screening tool for GAD, the GAD-7 has also proved to have

7 good sensitivity and specificity as a screener for panic disorder, social anxiety

8 disorder and post-traumatic stress disorder (Kroenke, Spitzer, Williams, Monahan, \&

9 Löwe, 2007). While the cut-off of 10 is optimal for detecting GAD, a cut-off of 8 has

10 been found to maximize sensitivity (77\%) and specificity (82\%) when detecting any

11 anxiety disorder (Kroenke et al., 2007). In this study, we used this cut-off ( $\geq 8$ ) to

12 provide an estimate of 'clinically significant anxiety'. Internal consistency for the

13 GAD-7 is excellent (Cronbach's $\alpha=.92$ ) and with a good test-retest reliability of $r=$.

14 83. In addition, convergent validity of the GAD-7 has been shown to be good, as

15 demonstrated by its correlations to the Beck Anxiety Inventory, $r=.72$, and the

16 anxiety dimension of SCL-90, $r=.74$ (Kroenke et al., 2010). Similarly to the PHQ-9,

17 the GAD-7 has been shown to be valid in the general population (Löwe et al., 2008).

18 The GAD-7 was chosen over other measures of anxiety due to its short length, free

19 availability, but also because of its possibility to screen for anxiety disorders in

20 general (Löwe et al., 2008).

\section{Generalized anxiety disorder}

22 To get an estimate of the prevalence of GAD, the Generalized Anxiety Disorder

23 Questionnaire-IV (GAD-Q-IV; Newman, Zuellig, \& Kachin, 2002) was used. It is a

24 questionnaire that mimics the structure of the DSM-IV diagnosis of GAD. The

25 instrument is a checklist that contains questions regarding excessive worry during the 
1 last 6 months. It also lets the participant register symptoms which are common among

2 patients with GAD, and lets the respondent give an account over his or her worry

3 areas or topics. The last question is about impact. A cut-off of 5.7 points (83\%

4 sensitivity and $89 \%$ specificity) on the GAD-Q-IV has been reported to indicate the

5 presence of GAD (Newman et al., 2002). Newman and colleagues (2002) proposed a

6 cut-off of 9.0 points (70\% sensitivity and 96\% specificity) to get larger certainty that

7 only persons with GAD are identified. In this study, respondents were instructed to

8 skip the rest of the GAD-Q-IV questions if they answered "no" to the first question

9 about experience of excessive worry which might have lead to existing GAD cases

10 not being identified. Skipped items were coded as zero. The cut-off score of 5.7 was

11 used in this study to provide an estimate of the prevalence of GAD.

\section{Health-related quality of life}

13 The EuroQol (EQ-5D; EuroQol Group, 1990) was used to assess health-related

14 quality of life (HRQoL). The instrument contains five dimensions: mobility, self-care,

15 usual activities, pain/discomfort and anxiety/depression where respondents rate their

16 current health on a three-point scale (EuroQol Group, 1990). In addition, there exists a

17 mean of converting the scores into a single index score, using a 'tariff' (Dolan, 1997).

18 This index score ranges from -0.594 to 1 , where full health is 1 and being dead is 0 (a

19 negative value represent a health condition considered worse than death). As there is

20 no tariff for Sweden, we used the tariff from the UK (Dolan, 1997) which is

21 commonly used in research from Sweden (Burström, Johannesson, \& Diderichsen, 22 2001). 


\section{Results}

2 Demographic data are illustrated in Table 1. There were some gender differences in

3 this data, as women (56.5\% had post-secondary education) were more educated than

4 men (45.3\% had post-secondary education). Women also had more experience of

5 treatments for worry and for psychiatric problems in general. Moreover, the rate of

6 experience of treatment for any psychiatric disorder was 52.2\% (95\% CI: 45.3 - 59.1;

$7 n=107)$ among individuals with clinically significant depression or anxiety $(n=205)$,

8 and $64.9 \%$ (95\% CI: $56.7-73.1 ; n=85)$ among those with major depression or GAD

$9 \quad(n=131)$.

\section{Prevalence of depression and anxiety disorders}

11 Prevalence rates are illustrated in Table 2 . The estimate of clinically significant

12 depression (PHQ-9 $\geq 10$ ) was about double (10.8\%) that of major depression (5.2\%),

13 using the diagnostic algorithm. Prevalence rates of anxiety disorders in general and of

14 GAD were $14.7 \%$ and $8.8 \%$, respectively. There were significant gender differences

15 for all estimates, as seen in Table 2. Average scores on the PHQ-9 and the GAD-7 in

16 the entire sample were $3.70(n=1240$; 95\% CI: $3.44-3.96)$ and $3.59(n=1266$; $95 \%$

17 CI: 3.36 - 3.81) respectively. On the PHQ-9, women scored higher, $4.21(n=683$;

18 95\% CI: 3.84 - 4.57), than men, $3.07(n=557 ; 95 \%$ CI: $2.72-3.42), t(1238)=4.29$,

$19 p<.001$. This was similar for the GAD-7, with women scoring 4.13 ( $n=704 ; 95 \%$

20 CI: $3.81-4.45)$ and men $2.91(n=562 ; 95 \%$ CI: $2.62-3.21), t(1264)=5.33, p<$.

21001.

\section{Prevalence rates using stricter definitions}

23 As a mean of having larger certainty that only individuals with the disorder were

24 identified, more strict cut-offs on the PHQ-9 $(\geq 15)$ and the GAD-Q-IV $(\geq 9.0)$ were 
1 employed. This resulted in prevalence rates of 4.4\% (95\% CI: $3.3-5.6)$ for

2 depression/depressive symptoms and 4.7\% (95\% CI: 3.5 - 5.8) for GAD.

\section{Prevalence of health-related quality of life problems}

6 The most prevalent HRQoL problems were reported in the dimension Pain/discomfort

7 where $38.9 \%$ of the participants who reported moderate or severe problems. This was

8 followed by the Anxiety/depression dimension where the corresponding figure was

$934.3 \%$. Prevalence of moderate/severe problems in the other dimensions were less

10 than 10\%. Gender differences were observed in the dimensions Usual activities and

11 Anxiety/depression. Details are presented in Table 3. The mean EQ-5D index value

12 was $0.84(0.83-0.85)$ for all participants $(n=1295)$. There was a significant

13 difference where men reported higher HRQoL (0.87; $n=572 ; 95 \%$ CI: $0.85-0.88)$

14 compared to women $(0.83 ; n=723$; 95\% CI: $0.81-0.84), t(1293)=3.46, p<.001$.

--INSERT TABLE 3--

\section{Comorbidity}

18 As seen in Table 2, the rate of comorbidity was 8.3\% (95\% CI: 6.8 - 9.9) and 3.1\%

19 (95\% CI: 2.1 - 4.1), when using the two different definitions. Put differently, among

20 those with clinically significant depression or anxiety, 48.3\% (100/207) had comorbid

21 depression and anxiety, while the same figure was 28.2\% (37/131) among those with

22 major depression or GAD. This is illustrated in further detail in Figure 1.

24 Patients with comorbid clinically significant depression and anxiety had higher

25 symptom severity, compared to those with a single diagnosis as measured by the 
1 PHQ-9 (14.91 compared to 8.21) and the GAD-7 (13.00 compared to 8.55). The same

2 was true for those with comorbid major depression and GAD (PHQ-9 score 18.81,

3 GAD-7 score 14.17), compared to those with either of the diagnoses (PHQ-9 score

4 10.55, GAD-7 score 9.99). All the differences were statistically significant (all $t$ 's $>$

5 5.13, all $\left.p^{\prime} \mathrm{s}<.001\right)$.

6

--INSERT FIGURE 1--

\section{Effects of depression, anxiety and their comorbidity on health-related quality of life}

11 HRQoL as measured by EQ-5D index values was consistently lower among

12 participants with disorders than those without: Clinically significant depression, 0.54

13 vs 0.88 ; clinically significant anxiety, 0.61 vs 0.88 ; major depression, 0.43 vs 0.87 ;

14 generalized anxiety disorder, 0.63 vs 0.86 . All differences were significant (all $t$ 's $>$

15 11.8, all $\left.p^{\prime} \mathrm{s}<.001\right)$.

17 Among participants with comorbid depression and anxiety, compared to those with a

18 single disorder, there were further differences in health-related quality of life. Mean

19 EQ-5D index values were $0.51(n=99 ; 95 \%$ CI: $0.45-0.58)$ and $0.71(n=105 ; 95 \%$

20 CI: $0.66-0.75$ ) for comorbid/not comorbid clinically significant depression and

21 anxiety, and $0.45(n=35$; 95\% CI: $0.36-0.54)$ and $0.63(n=90$; 95\% CI: $0.57-$

22 0.69) for participant with comorbid major depression and GAD compared to those

23 with a single diagnosis. These differences were significant (both $t^{\prime}$ s $>2.96$, both $p$ 's $<$.

24 01). Significant differences due to comorbidity were also observed in EQ-5D

25 dimensions Usual activities and Anxiety/depression for both definitions of 
1 comorbidity, and also for Pain/discomfort for clinically depressed or anxious

2 participants. Details of these analyses are presented in Table 4.

\section{Discussion}

6 This study suggest that, at any given time point, around $17.2 \%$ of the Swedish general

7 population are experiencing clinically significant depression (10.8\%) or anxiety

8 (14.7\%) that likely affect their daily lives. Around 5.2\% experienced a current major

9 depressive episode and 8.8\% had generalized anxiety disorder. Half of those with

10 either clinically significant depression or anxiety also had a comorbid disorder.

11 Comorbidity among disorders was associated with higher symptom severity and lower

12 health-related quality of life.

14 Between half and two thirds of participants with a disorder had treatment experiences.

15 This is in line with what was found in the US National Comorbidity Survey

16 Replication (NCS-R), where only 51.6\% (95\% CI: 46.1 - 57.2) of 12-month cases

17 received health care treatment for depression, and where only 21.7\% (95\% CI: 18.1 -

18 25.2) were adequately treated (Kessler et al., 2003). Furthermore, there are data from

19 a community survey in Finland, where the use of health services for mental health

20 was investigated (Hämäläinen, Isometsä, Sihvo, Pirkola, \& Kiviruusu, 2008). Among

21 individuals with depression, anxiety disorders, or both, only 34\%, 36\%, and 59\% used

22 health services, respectively. In summary, data from the present study are in line with

23 treatment consumption studies from other countries, and indicate that depression and

24 anxiety disorders are undertreated conditions also in the Swedish general population. 
1 This study estimated the point prevalence of major depression in Sweden to be 5.2\%.

2 This figure is in line with the 12 month prevalence rates from the US (6.7\%, 95\% CI:

3 6.1 - 7.3; Kessler, Chiu, et al., 2005), Australia (6.3\%, 95\% CI: 5.7 - 6.9 ; Andrews,

4 Henderson, \& Hall, 2001) and the Netherlands (5.8\%, 95\% CI: 5.2 - 6.4; Bijl, Ravelli,

5 \& Van Zessen, 1998). The figure $5.2 \%$ is also close to the original estimate of $4.7 \%$,

6 from the 1957 population in the Lundby study (Rorsman et al., 1990). When using a

7 stricter definition (only 5\% risk of detecting false positives), $4.4 \%$ of individuals were

8 identified as having major depression. This and other prevalence estimates provide

9 further indications that $5.2 \%$ is indeed a valid estimate of current point prevalence of

10 major depression in Sweden, and therefore complements epidemiological research

11 from the Lundby study (Rorsman et al., 1990) and the national Swedish Twin

12 Registry (Kendler et al., 2006).

14 A cut-off of PHQ-9 $\geq 10$ was used to identify clinically significant depression, and

15 was found among $10.8 \%$ of individuals in this study. This rate is similar or somewhat

16 higher compared to previous estimates using the same definition in the US (8.6\%;

17 Kroenke et al., 2009) and in Canada (8.4\%; Patten \& Schopflocher, 2009). Our result

18 on clinically significant depression seems to mirror estimates of 'any mood disorder',

19 e.g. from the NCS-R (Kessler et al., 2005) where this was found among 9.5\% (95\%

20 CI: $8.7-10.3)$.

22 We found the point prevalence of clinically significant anxiety in Sweden to be

$2314.7 \%$, using the definition of GAD-7 $\geq 8$. This seems similar or somewhat higher

24 than previous estimates from Germany (12.1\%; Löwe et al., 2008). The mean GAD-7

25 score in the present study (3.59, 95\% CI: 3.36 - 3.81) was higher than the average 
1 score from the German population study (2.95, 95\% CI: 2.85 - 3.04) (Löwe et al.,

2 2008). The rate of clinically significant anxiety found in this study can be compared

3 with that of $18.1 \%$ for any anxiety disorder from the NCS-R (Kessler et al., 2005).

4 Importantly, the GAD-7 is known only to identify GAD, social phobia, panic disorder

5 and PTSD (Kroenke et al., 2007). The NCS-R estimate did also include specific

6 phobia, which was found in 8.7\% of US 12 month cases (Kessler et al., 2005). In

7 conclusion, our estimates of prevalence of general anxiety seem comparable to other

8 epidemiological studies.

10 Our estimate of GAD prevalence, 8.8\%, using the definition of GAD-Q-IV $\geq 5.7$

11 (Newman et al., 2002), seem significantly higher than in other epidemiological studies

12 from e.g. US (3.1\%; Kessler, Chiu, et al., 2005) and Australia (2.6\%; Andrews et al.,

13 2001). The stricter definition of GAD-Q-IV $\geq 9.0$ (95\% specificity) gave an estimate

14 of $4.7 \%$, that indicates that the true prevalence in Sweden is probably at least that

15 high. Importantly, as noted by Kessler and colleagues (Kessler, Walters, \& Wittchen,

16 2004) in a review of the epidemiology of GAD, there are uncertainties about the basic

17 epidemiological characteristics of GAD that could lead to underestimates of the true

18 prevalence of GAD in the general population. There are known complicating factors

19 due to changing DSM criteria over time, and the fact that DSM definitions of GAD

20 seem to exclude a substantial amount of individuals with chronic worry, tension and

21 nervousness (Kessler et al., 2004). Kessler et al. (2004) argued that the true current

22 prevalence of GAD in the community could actually be as high as 5 to $8 \%$. Our study

23 has provided indications that the prevalence of GAD in the Swedish general

24 population probably is within this interval.

25

PeerJ reviewing PDF | (v2013:05:526:2:0:NEW 14 Jun 2013) 
1 The EQ-5D index value obtained in this study (0.84) was identical to that obtained in

2 a study that validates the EQ-5D in the Swedish general population (Burström et al.,

3 2001). Absence of reported confidence intervals in (Burström et al., 2001) prohibits a

4 more detailed comparison, but when comparing HRQoL data from this study (Table

5 3) to Swedish normative data, no dimension differ more than $5.4 \%$. This gives

6 indications that HRQoL data collected in this study are overall similar to previous

7 collected data from Sweden.

9 There are methodological limitations that need to be considered. In this study, we

10 used self-report measures, rather than a diagnostic interview, such as the SCID (First,

11 Gibbons, Spitzer, \& Williams, 1997) or the CIDI (Robins et al., 1988). While the

12 measures used in this study are thoroughly validated (Kroenke et al., 2001; Newman

13 et al., 2002; Spitzer et al., 2006), also for use in the general population (Kroenke et

14 al., 2009; Löwe et al., 2008) we still consider this as a limitation. A further limitation

15 is that with the data collected on educational level it was impossible to interpret

16 whether a respondent had a certain education ongoing or completed. This makes the

17 collected rate of 52\% with post-secondary education hard to interpret. According to

18 national statistics from Statistics Sweden (Statistics Sweden, 2010), this figure is

$1935.5 \%$. This indicates that our data on educational level may not be valid. A final

20 important limitation of this study is the response rate of $44.3 \%$, as for example

21 compared to (Kessler et al., 2005) who had 70.9\% response rate. Among community

22 surveys using the PHQ-9, our response rate is low in comparison to 64.5\% (Martin et

23 al., 2006), but higher than in Pirkis et al. (2009) who reported 28.6\%. This could have

24 biased the prevalence estimates in this study. Importantly, there are indications that

25 individuals with mental illness might be more reluctant than others to participate in 
1 mental health surveys (Allgulander, 1989; Eaton, Anthony, Tepper, \& Dryman,

2 1992). Based on this, prevalence estimates of depression and anxiety disorders in this

3 study would be underestimated, if biased. Future research with a similar design to this

4 study should make use of established strategies to increase the response rate, for

5 example by using monetary incentives, using pre-notification and by having a shorter

6 questionnaire (Edwards et al., 2007).

8 Some implications of this study are discussed as follows. The results from this study

9 complements and corroborates earlier findings from Sweden and other countries by

10 providing up-to-date prevalence rates. The fact that $17.2 \%$ of the population suffer

11 from a clinically significant depression or anxiety condition calls for further efforts

12 regarding prevention and treatment. This includes both novel and more established

13 intervention alternatives. The use of the Internet is one way of enabling

14 evidence-based interventions to reach larger proportions of the general population

15 (Muñoz, 2010). Internet-based psychological treatments seem to be a promising and

16 feasible treatment alternative that may increase availability of evidence-based

17 treatments for psychiatric disorders such as depression and anxiety (Andersson, 2009;

18 Hedman, Ljótsson, \& Lindefors, 2012). Future challenges include establishing means

19 of delivering such treatment in regular health-care (Hedman et al., 2013), but also on

20 national level with preserved efficacy (Calear, Christensen, Mackinnon, Griffiths, \&

21 O’Kearney, 2009).

23 A further implication regards the significant amount of comorbidity between

24 depression and anxiety disorders identified in this study (see Figure 1). The large

25 overlap calls for alternatives to disorder-focused assessments and treatments that 
1 address transdiagnostic factors, such as emotion regulation deficits (Aldao,

2 Nolen-Hoeksema, \& Schweizer, 2010; Mennin, McLaughlin, \& Flanagan, 2009). This

3 could potentially enable assessment that is grounded in experimental research and that

4 provide an opportunity for treatment alternatives that target these processes (e.g.,

5 Farchione et al., 2012; Johansson, Hesser, Ljótsson, Frederick, \& Andersson, 2012;

6 McEvoy, Nathan, \& Norton, 2009). Hence, transdiagnostic treatment approaches

7 seem as an important step in addressing psychiatric comorbidity.

9 Finally, there are indications that positive mental health likely is more than absence of 10 psychiatric symptoms (Huppert \& Whittington, 2003). For example, the absence of

11 positive emotions have been found to be a better predictor of mortality than the

12 presence of psychological symptoms (Huppert \& Whittington, 2003). Also, there are

13 indications that disability and lack of social roles are important determinants of

14 psychological symptoms, but have less influence on positive well-being. Similarly,

15 paid employment has been found to be an important determinant of positive

16 well-being but it seem to have little influence on psychiatric symptoms (Huppert \&

17 Whittington, 2003). Therefore, future epidemiological research that aims to

18 understand the whole picture of human health seems to require assessments that go

19 beyond mere symptoms (Cloninger, 2006; Huppert \& Whittington, 2003).

\section{Conclusions}

21 The findings reported here provide updated prevalence rates from the Swedish general

22 population. Depression, anxiety and their comorbidity are undertreated conditions that

23 are associated with lower quality of life. This study adds to existing evidence that

24 these conditions are clearly major health problems in Sweden, that need further efforts

25 regarding preventive and treatment interventions. 


\section{Acknowledgements}

2 We would like to thank Elinore Hellqvist for help during the data collection.

\section{References}

4 Albert, U., Rosso, G., Maina, G., \& Bogetto, F. (2008). Impact of anxiety disorder

5 comorbidity on quality of life in euthymic bipolar disorder patients: differences

6 between bipolar I and II subtypes. Journal of affective disorders, 105, 297-303.

7 Aldao, A., Nolen-Hoeksema, S., \& Schweizer, S. (2010). Emotion-regulation strategies across psychopathology: A meta-analytic review. Clinical psychology

Allgulander, C. (1989). Psychoactive drug use in a general population sample,

Sweden: correlates with perceived health, psychiatric diagnoses, and mortality in an automated record-linkage study. American journal of public health, 79,

14 Allgulander, C., \& Nilsson, B. (2003). Var fjärde patient lider av angest och depression [A nationwide study in primary health care: One out of four patients suffers from anxiety and depression]. Läkartidningen, 100, 832-8.

Andersson, G. (2009). Using the Internet to provide cognitive behaviour therapy. Behaviour research and therapy, 47, 175-80.

19 Andrews, G., Henderson, S., \& Hall, W. (2001). Prevalence, comorbidity, disability

20 and service utilisation. Overview of the Australian National Mental Health

21 Survey. The British journal of psychiatry: the journal of mental science, 178, $22 \quad 145-53$.

23 Baumeister, H., \& Härter, M. (2007). Prevalence of mental disorders based on general 24 population surveys. Social psychiatry and psychiatric epidemiology, 42, 537-46. 
1 Beck, A. T., Steer, R. A., \& Brown, G. (1996). Beck depression inventory-II: Manual. Boston: Harcourt Brace.

3 Bijl, R. V, Ravelli, A., \& Van Zessen, G. (1998). Prevalence of psychiatric disorder in

7 Burström, K., Johannesson, M., \& Diderichsen, F. (2001). Swedish population

11 Calear, A. L., Christensen, H., Mackinnon, A., Griffiths, K. M., \& O’Kearney, R.

22 Carter, R. M., Wittchen, H. U., Pfister, H., \& Kessler, R. C. (2001). One-year

23 prevalence of subthreshold and threshold DSM-IV generalized anxiety disorder

24 in a nationally representative sample. Depression and anxiety, 13, 78-88. 
1 Cloninger, C. R. (2006). The science of well-being: an integrated approach to mental health and its disorders. World psychiatry: official journal of the World Psychiatric Association (WPA), 5, 71-6.

4 Dolan, P. (1997). Modeling valuations for EuroQol health states. Medical care, 35, $5 \quad 1095-108$.

6 Dum, M., Pickren, J., Sobell, L. C., \& Sobell, M. B. (2008). Comparing the BDI-II 7 and the PHQ-9 with outpatient substance abusers. Addictive behaviors, 33, $8 \quad 381-7$.

9 Eaton, W. W., Anthony, J. C., Tepper, S., \& Dryman, A. (1992). Psychopathology and attrition in the epidemiologic catchment area surveys. American journal of epidemiology, 135, 1051-9.

12 Ebmeier, K. P., Donaghey, C., \& Steele, J. D. (2006). Recent developments and current controversies in depression. Lancet, 367, 153-67.

14 Edwards, P., Roberts, I., Clarke, M., DiGuiseppi, C., Pratap, S., Wentz, R., Kwan, I., \& Cooper, R. (2007). Methods to increase response rates to postal questionnaires. Cochrane database of systematic reviews, MR000008.

17 EuroQol Group. (1990). EuroQol--a new facility for the measurement of health-related quality of life. The EuroQol Group. Health policy (Amsterdam, Netherlands), 16, 199-208.

20 Farchione, T. J., Fairholme, C. P., Ellard, K. K., Boisseau, C. L., Thompson-Hollands, 21 J., Carl, J. R., Gallagher, M. W., \& Barlow, D. H. (2012). Unified protocol for 22 transdiagnostic treatment of emotional disorders: a randomized controlled trial. 23 Behavior therapy, 43, 666-78. 
1 First, M. B., Gibbons, R. D., Spitzer, R. L., \& Williams, J. B. (1997). SCID-I: American Psychiatric Press.

4 Furmark, T., Tillfors, M., Everz, P., Marteinsdottir, I., Gefvert, O., \& Fredrikson, M.

8 Hedman, E., Ljótsson, B., \& Lindefors, N. (2012). Cognitive behavior therapy via the Internet: a systematic review of applications, clinical efficacy and cost-effectiveness. Expert review of pharmacoeconomics \& outcomes research, $12,745-64$.

12 Hedman, E., Ljótsson, B., Rück, C., Bergström, J., Andersson, G., Kaldo, V., Jansson, 13 L., Andersson, E., Blom, K., El Alaoui, S., Falk, L., Ivarsson, J., Nasri, B., Rydh, 14 S., \& Lindefors, N. (2013). Effectiveness of Internet-based cognitive behaviour 15 therapy for panic disorder in routine psychiatric care. Acta psychiatrica 16 Scandinavica.

17 Huppert, F. A., \& Whittington, J. E. (2003). Evidence for the independence of positive and negative well-being: implications for quality of life assessment.

20 Hämäläinen, J., Isometsä, E., Sihvo, S., Pirkola, S., \& Kiviruusu, O. (2008). Use of 21 health services for major depressive and anxiety disorders in Finland.

22 Depression and anxiety, 25, 27-37.

23 Johansson, R., Hesser, H., Ljótsson, B., Frederick, R. J., \& Andersson, G. (2012).

24 Transdiagnostic, affect-focused, psychodynamic, guided self-help for depression 
and anxiety through the internet: study protocol for a randomised controlled trial. BMJ open, 2.

3 Kendler, K. S., Gatz, M., Gardner, C. O., \& Pedersen, N. L. (2006). A Swedish national twin study of lifetime major depression. The American journal of psychiatry, 163, 109-14.

6 Kessler, R. C., Berglund, P., Demler, O., Jin, R., Koretz, D., Merikangas, K. R., Rush,

Kroenke, K., Spitzer, R. L., \& Williams, J. B. (2001). The PHQ-9: validity of a brief 
1 Kroenke, K., Spitzer, R. L., Williams, J. B. W., \& Löwe, B. (2010). The Patient Health Questionnaire Somatic, Anxiety, and Depressive Symptom Scales: a systematic review. General hospital psychiatry, 32, 345-59.

4 Kroenke, K., Spitzer, R. L., Williams, J. B. W., Monahan, P. O., \& Löwe, B. (2007).

5 Anxiety disorders in primary care: prevalence, impairment, comorbidity, and 6 detection. Annals of internal medicine, 146, 317-25.

7 Kroenke, K., Strine, T. W., Spitzer, R. L., Williams, J. B. W., Berry, J. T., \& Mokdad,

8 A. H. (2009). The PHQ-8 as a measure of current depression in the general 9 population. Journal of affective disorders, 114, 163-73.

10 Löwe, B., Decker, O., Müller, S., Brähler, E., Schellberg, D., Herzog, W., \& Herzberg, P. Y. (2008). Validation and standardization of the Generalized

2
Anxiety Disorder Screener (GAD-7) in the general population. Medical care, 46,

Martin, A., Rief, W., Klaiberg, A., \& Braehler, E. (2006). Validity of the Brief Patient Health Questionnaire Mood Scale (PHQ-9) in the general population. General hospital psychiatry, 28, 71-7.

7 McEvoy, P., Nathan, P., \& Norton, P. (2009). Efficacy of transdiagnostic treatments:

18 A review of published outcome studies and future research directions. Journal of cognitive psychotherapy: An international quarterly, 23, 20-33.

20 Mennin, D. S., McLaughlin, K. A., \& Flanagan, T. J. (2009). Emotion regulation

21 deficits in generalized anxiety disorder, social anxiety disorder, and their

22 co-occurrence. Journal of anxiety disorders, 23, 866-71.

23 Munk-Jørgensen, P., Allgulander, C., Dahl, A. A., Foldager, L., Holm, M.,

24 Rasmussen, I., Virta, A., Huuhtanen, M.-T., \& Wittchen, H.-U. (2006). 
1 Prevalence of generalized anxiety disorder in general practice in Denmark,

2 Finland, Norway, and Sweden. Psychiatric services (Washington, D.C.), 57,

3 1738-44.

4 Muñoz, R. F. (2010). Using evidence-based internet interventions to reduce health

5 disparities worldwide. Journal of medical Internet research, 12, e60.

6 Newman, M., Zuellig, A., \& Kachin, K. (2002). Preliminary reliability and validity of

7 the Generalized Anxiety Disorder Questionnaire-IV: A revised self-report

8 diagnostic measure of generalized anxiety disorder. Behavior Therapy.

9 Patten, S. B., \& Schopflocher, D. (2009). Longitudinal epidemiology of major

10 depression as assessed by the Brief Patient Health Questionnaire (PHQ-9).

11 Comprehensive psychiatry, 50, 26-33.

12 Pirkis, J., Pfaff, J., Williamson, M., Tyson, O., Stocks, N., Goldney, R., Draper, B.,

13 Snowdon, J., Lautenschlager, N., \& Almeida, O. P. (2009). The community

14 prevalence of depression in older Australians. Journal of affective disorders, $15 \quad 115,54-61$.

16 Robins, L. N., Wing, J., Wittchen, H. U., Helzer, J. E., Babor, T. F., Burke, J.,

17 Farmer, A., Jablenski, A., Pickens, R., \& Regier, D. A. (1988). The Composite

18 International Diagnostic Interview. An epidemiologic Instrument suitable for use

19 in conjunction with different diagnostic systems and in different cultures.

20 Archives of general psychiatry, 45, 1069-77.

21 Rorsman, B., Gräsbeck, A., Hagnell, O., Lanke, J., Ohman, R., Ojesjö, L., \&

22 Otterbeck, L. (1990). A prospective study of first-incidence depression. The

23 Lundby study, 1957-72. The British journal of psychiatry: the journal of mental

24 science, 156, 336-42. 
1 Saarni, S. I., Suvisaari, J., Sintonen, H., Pirkola, S., Koskinen, S., Aromaa, A., \& Lönnqvist, J. (2007). Impact of psychiatric disorders on health-related quality of life: general population survey. The British journal of psychiatry: the journal of mental science, 190, 326-32.

5 Schoevers, R. A., Deeg, D. J. H., Van Tilburg, W., \& Beekman, A. T. F. (2005).

6 Depression and generalized anxiety disorder: co-occurrence and longitudinal

7 patterns in elderly patients. The American journal of geriatric psychiatry: official journal of the American Association for Geriatric Psychiatry, 13, 31-9.

9 Sherbourne, C. D., Sullivan, G., Craske, M. G., Roy-Byrne, P., Golinelli, D., Rose, R.

$$
\text { D., Chavira, D. A., Bystritsky, A., \& Stein, M. B. (2010). Functioning and }
$$

1
disability levels in primary care out-patients with one or more anxiety disorders.

13 Spitzer, R. L., Kroenke, K., Williams, J. B. W., \& Löwe, B. (2006). A brief measure

14 for assessing generalized anxiety disorder: the GAD-7. Archives of internal 15 medicine, $166,1092-7$.

16 Statistics Sweden. (2010). Educational level of Sweden 2010 by age.

17 Titov, N., Dear, B. F., McMillan, D., Anderson, T., Zou, J., \& Sunderland, M. (2011).

18 Psychometric comparison of the PHQ-9 and BDI-II for measuring response

19 during treatment of depression. Cognitive Behaviour Therapy, 40, 126-136.

20 Von Elm, E., Altman, D. G., Egger, M., Pocock, S. J., Gøtzsche, P. C., \&

21 Vandenbroucke, J. P. (2007). The Strengthening the Reporting of Observational

22 Studies in Epidemiology (STROBE) statement: guidelines for reporting

23 observational studies. PLoS medicine, 4, e296. 


\section{Figures}

\section{Figure 1}

3 A: Prevalence rates of clinically significant depression (134/1240), clinically

4 significant anxiety (186/1266) and their comorbidity (100/1201). Also illustrated are

5 clinically significant depression without anxiety (30/1245) and clinically significant

6 anxiety without depression (77/1261).

7

8 B: Prevalence rates of current major depression (65/1240), GAD (112/1269) and their

9 comorbidity (37/1195). Furthermore, current major depression without GAD

10 (26/1266) and GAD without major depression (71/1267).

11

12 Note: As the number of respondents were different for various measures, different

13 prevalence rates in the figure may not add up to exactly the same figure as that given.

14

15 Abbreviations: GAD, Generalized anxiety disorder. 


\section{Table $\mathbf{1}_{\text {(on next page) }}$}

Demographic description of the participants. 


\section{Table 1}

Demographic description of the participants.

\begin{tabular}{|c|c|c|c|c|c|}
\hline & & Male & Female & Total & Test statistics \\
\hline \multirow[t]{2}{*}{ Age } & Mean (SD) $\stackrel{\varrho}{=}$ & $46.6(14.3)$ & $45.9(14.6)$ & $46.2(14.5)$ & $t(1193)=0.868, p=.39$ \\
\hline & $\operatorname{Min-Max} \frac{\stackrel{\mathcal{D}}{\rightleftharpoons}}{\frac{\tau}{\sigma}}$ & $18-68$ & $18-68$ & $18-68$ & \\
\hline \multirow[t]{2}{*}{ Marital status } & Married or $\overline{\text { so }}$-habiting & $424(72.9 \%)$ & $549(74.2 \%)$ & $973(73.6 \%)$ & $\begin{array}{l}\chi 2(N=1322, d f=1)=0.30 \\
p=.58\end{array}$ \\
\hline & Single $\quad \stackrel{3}{-\frac{d}{>}}$ & $158(27.1 \%)$ & $191(25.8 \%)$ & $349(26.4 \%)$ & \\
\hline Having children & Yes $\frac{0}{\square}$ & $436(74.7 \%)$ & $572(76.8 \%)$ & $1008(75.8 \%)$ & $\begin{array}{l}\chi 2(N=1329, d f=1)=0.80 \\
p=.37\end{array}$ \\
\hline \multirow[t]{4}{*}{ Educational level } & Primary (b) & $98(18.0 \%)$ & $99(13.9 \%)$ & $197(15.7 \%)$ & $\begin{array}{l}\chi 2(N=1256, d f=3)=15.7 \\
p<.001\end{array}$ \\
\hline & Secondary & 199 (36.6\%) & $211(29.6 \%)$ & $410(32.6 \%)$ & \\
\hline & Post-secondary $0-2,9$ years & $90(16.6 \%)$ & $144(20.2 \%)$ & $234(18.6 \%)$ & \\
\hline & Post-secondary $3+$ years & $156(28.7 \%)$ & $259(36.3 \%)$ & $415(33.0 \%)$ & \\
\hline $\begin{array}{l}\text { Experiences of treatment } \\
\text { for worrying }\end{array}$ & Yes & $76(13.1 \%)$ & $164(22.3 \%)$ & $240(18.3 \%)$ & $\begin{array}{l}\chi 2(N=1314, d f=1)=18.1 \\
p<.001\end{array}$ \\
\hline $\begin{array}{l}\text { Experiences of any } \\
\text { treatment for psychiatric } \\
\text { problems }\end{array}$ & Yes & $91(15.7 \%)$ & $197(26.7 \%)$ & $288(21.9 \%)$ & $\begin{array}{l}\chi 2(N=1315, d f=1)=22.9 \\
p<.001\end{array}$ \\
\hline
\end{tabular}




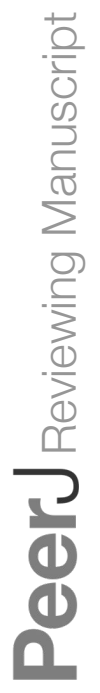




\section{Table $2_{\text {(on next page) }}$}

Prevalence estimates. 95\% confidence intervals are given in parenthesis.

Abbreviations: PHQ-9: 9-item Patient Health Questionnaire Depression Scale; GAD-7: 7-item Generalized Anxiety Disorder Scale; GAD-Q-IV: Generalized Anxiety Disorder Questionnaire-IV. 


\section{Table 2}

Prevalence estimates. 95\% confidence intervals are given in parenthesis.

\begin{tabular}{|c|c|c|c|c|c|}
\hline & Definition & Male & Female & Total & Test statistics \\
\hline \multicolumn{6}{|c|}{ Clinically significant depression or anxiety } \\
\hline Depression & PHQ-9 $\geq 10$ & $8.3 \%(6.0-10.5)$ & $12.9 \%(10.4-15.4)$ & $10.8 \%(9.1-12.5)$ & $\begin{array}{l}\chi 2(N=1240, d f= \\
1)=6.8, p<.01\end{array}$ \\
\hline Anxiety & GAD-7 $\geq 8$ & $10.7 \%(8.1-13.2)$ & $17.9 \%(15.1-20.7)$ & $14.7 \%(12.7-16.6)$ & $\begin{array}{l}\chi 2(N=1266, d f= \\
1)=13.0, p<.001\end{array}$ \\
\hline $\begin{array}{l}\text { Comorbid } \\
\text { depression and } \\
\text { anxiety }\end{array}$ & $\begin{array}{l}\text { PHQ-9 } \geq 10 \text { and } \\
\text { GAD-7 } \geq 8\end{array}$ & $5.7 \%(3.8-7.7)$ & $10.5 \%(8.1-12.8)$ & $8.3 \%(6.8-9.9)$ & $\begin{array}{l}\chi 2(N=1201, d f= \\
1)=8.8, p<.01\end{array}$ \\
\hline Any disorder & $\begin{array}{l}\text { PHQ-9 } \geq 10 \text { and } / \mathrm{C} \\
\text { GAD-7 } \geq 8\end{array}$ & $13.3 \%(10.4-16.1)$ & $20.5 \%(17.4-23.6)$ & $17.2 \%(15.1-19.4)$ & $\begin{array}{l}\chi 2(N=1201, d f= \\
1)=10.8, p<.001\end{array}$ \\
\hline \multicolumn{6}{|c|}{ Major depression or Generalized anxi tis disorder } \\
\hline Major depression & PHQ-9 algorithm & $3.4 \%(1.9-4.9)$ & $6.7 \%(4.8-8.6)$ & $5.2 \%(4.0-6.5)$ & $\begin{array}{l}\chi 2(N=1240, d f= \\
1)=6.82, p<.01\end{array}$ \\
\hline GAD & GAD-Q-IV $\geq 5.7$ & $6.6 \%(4.6-8.7)$ & $10.6 \%(8.3-12.8)$ & $8.8 \%(7.3-10.4)$ & $\begin{array}{l}\chi 2(N=1269, d f= \\
1)=6.13, p<.05\end{array}$ \\
\hline $\begin{array}{l}\text { Comorbid major } \\
\text { depression and } \\
\text { GAD }\end{array}$ & $\begin{array}{l}\text { PHQ-9 algorithm } \\
\text { and GAD-Q-IV } \geq \\
5.7\end{array}$ & $2.2 \%(1.0-3.5)$ & $3.8 \%(2.3-5.3)$ & $3.1 \%(2.1-4.1)$ & $\begin{array}{l}\chi 2(N=1195, d f= \\
1)=2.4, n s\end{array}$ \\
\hline Any disorder & $\begin{array}{l}\text { PHQ-9 algorithm } \\
\text { and/or GAD-Q-IV } \geq \\
5.7\end{array}$ & $8.0 \%(5.7-10.3)$ & $13.4 \%(10.8-16.0)$ & $11.0 \%(9.2-12.7)$ & $\begin{array}{l}\chi 2(N=1195, d f= \\
1)=8.8, p<.01\end{array}$ \\
\hline
\end{tabular}


Abbreviations: PHQ-9: 9-item Patient Health Questionnaire Depression Scale; GAD-7: 7-item Generalized Anxiety Disorder Scale; GAD-Q-IV: Generalized Anxiety Disorder Questionnaire-IV.

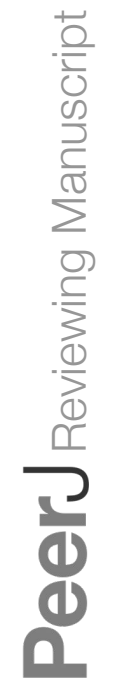




\section{Table 3 (on next page)}

EuroQol (EQ-5D) distribution (percentage) of respondents reporting no problems, moderate or severe problems in different dimensions, by gender. 


\section{Table 3}

EuroQol (EQ-5D) distribution (percentage) of respondents reporting no problems, moderate or severe problems in different dimensions, by gender.

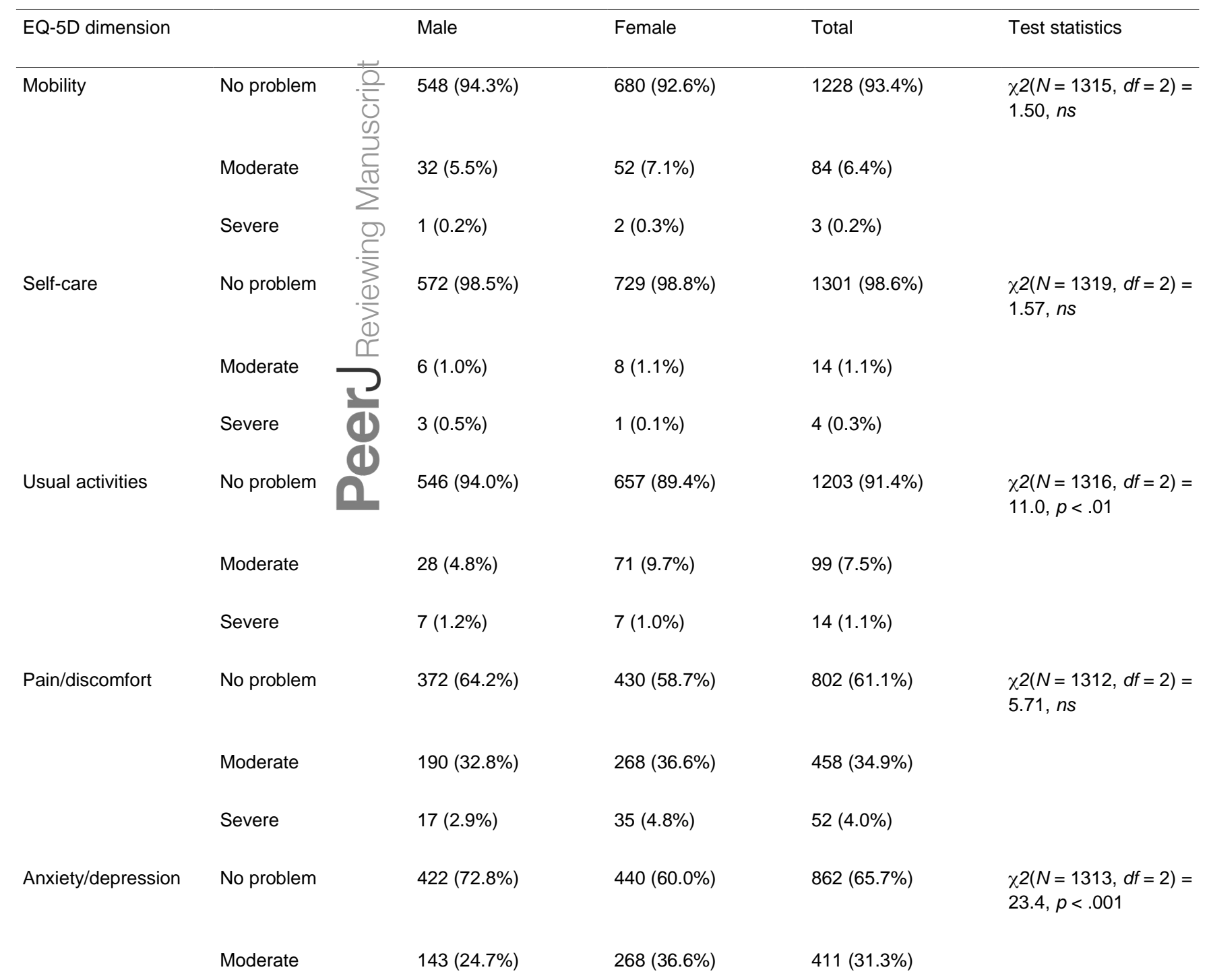




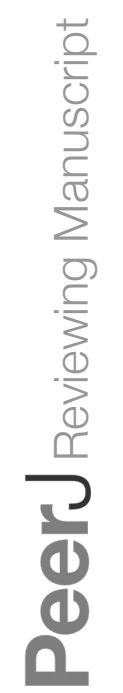




\section{Table 4(on next page)}

EuroQol (EQ-5D) distribution (percentage) among participant with depression and anxiety disorders, by comorbidity and single diagnosis.

Note: 'Clinically significant depression and anxiety' was defined as having a score of at least 10 on either or both of the PHQ-9 and the GAD-7. 'Major depression or GAD' was defined as either having a diagnosis of major depression using the PHQ-9 algorithm, or having a score of at least 5.7 on the GAD-Q-IV, or both.

Abbreviations: GAD: Generalized Anxiety Disorder. PHQ-9: 9-item Patient Health Questionnaire Depression Scale; GAD-7: 7-item Generalized Anxiety Disorder Scale; GAD-Q-IV: Generalized Anxiety Disorder Questionnaire-IV. 


\section{Table 4}

EuroQol (EQ-5D) distribution (percentage) among participant with depression and anxiety disorders, by comorbidity and single diagnosis.

\begin{tabular}{|c|c|c|c|c|c|c|c|c|c|}
\hline \multirow{2}{*}{ EQ-5D dimension } & & \multicolumn{4}{|c|}{ Clinically significant depression or anxiety } & \multicolumn{4}{|c|}{ Major depression or GAD } \\
\hline & & Comôbidity & No comorbidity & Total & Test statistics & Comorbidity & No comorbidity & Total & Test statistics \\
\hline \multirow[t]{3}{*}{ Mobility } & No problem & 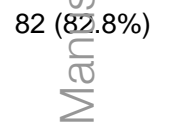 & $95(88.8 \%)$ & $177(85.9 \%)$ & $\begin{array}{l}\chi 2(N=206, d f= \\
2)=1.57, n s\end{array}$ & $32(88.9 \%)$ & 78 (83.9\%) & $110(85.3 \%)$ & $\begin{array}{l}\chi 2(N=129, d f=2)= \\
60, n s\end{array}$ \\
\hline & Moderate & 16 (10; $2 \%)$ & $11(10.3 \%)$ & $27(13.1 \%)$ & & $4(11.1 \%)$ & $13(14.0 \%)$ & $17(13.2 \%)$ & \\
\hline & Severe & $1(1.9 \%)$ & $1(0.9 \%)$ & $2(1.0 \%)$ & & $0(0.0 \%)$ & $2(2.2 \%)$ & $2(1.6 \%)$ & \\
\hline \multirow[t]{3}{*}{ Self-care } & No problem & $92(\vartheta 2.0 \%)$ & $103(97.2 \%)$ & $195(94.7 \%)$ & $\begin{array}{l}\chi 2(N=206, d f= \\
2)=3.23, n s\end{array}$ & $34(91.9 \%)$ & $89(95.7 \%)$ & $123(94.6 \%)$ & $\begin{array}{l}\chi 2(N=130, d f=2)= \\
.82, n s\end{array}$ \\
\hline & Moderate & $7((0 \%)$ & $2(1.9 \%)$ & $9(4.4 \%)$ & & $2(5.4 \%)$ & $3(3.2 \%)$ & $5(3.8 \%)$ & \\
\hline & Severe & $1 \%$ & $1(0.9 \%)$ & $2(1.0 \%)$ & & $1(2.7 \%)$ & $1(1.1 \%)$ & $2(1.5 \%)$ & \\
\hline \multirow[t]{3}{*}{ Usual activities } & No problem & $57(57.0 \%)$ & 87 (81.3\%) & $144(69.6 \%)$ & $\begin{array}{l}\chi 2(N=207, d f= \\
2)=15.4, p< \\
001\end{array}$ & 15 (40.5\%) & 69 (74.2\%) & $8.4(64.6 \%)$ & $\begin{array}{l}\chi 2(N=130, d f=2)= \\
15.6, p<.001\end{array}$ \\
\hline & Moderate & 37 (37.0\%) & $15(14.0 \%)$ & $52(25.1 \%)$ & & $20(54.1 \%)$ & 18 (19.4\%) & 38 (29.2\%) & \\
\hline & Severe & $6(6.0 \%)$ & $5(4.7 \%)$ & $11(5.3 \%)$ & & $2(5.4 \%)$ & $6(6.5 \%)$ & 8 (6.2\%) & \\
\hline \multirow[t]{3}{*}{ Pain/discomfort } & No problem & 25 (25.0\%) & 48 (45.3\%) & 73 (35.4\%) & $\begin{array}{l}\chi 2(N=206, d f= \\
2)=10.7, p< \\
01\end{array}$ & $10(27.0 \%)$ & 37 (39.8\%) & 47 (36.2\%) & $\begin{array}{l}\chi 2(N=130, d f=2)= \\
2.3, n s\end{array}$ \\
\hline & Moderate & 57 (57.0\%) & 49 (46.2\%) & $106(51.5 \%)$ & & 22 (59.5\%) & 42 (45.2\%) & 64 (49.2\%) & \\
\hline & Severe & $18(18.0 \%)$ & $9(8.5 \%)$ & 27 (13.1\%) & & $5(13.5 \%)$ & $14(15.1 \%)$ & $19(14.6 \%)$ & \\
\hline Anxiety/depressio & No problem & $2(2.0 \%)$ & $13(12.1 \%)$ & 15 (7.2\%) & $\chi 2(N=207, d f=$ & $0(0.0 \%)$ & $5(5.3 \%)$ & $5(3.8 \%)$ & $\chi 2(N=130, d f=2)=$ \\
\hline
\end{tabular}




$\begin{array}{lllllr}\text { Moderate } & 66(66.0 \%) & 88(82.2 \%) & 154(74.4 \%) & 18(50.0 \%) & 76(80.9 \%) \\ \text { Severe } & 32(32.0 \%) & 6(5.6 \%) & 38(18.4 \%) & 18(50.0 \%) & 13(13.8 \%)\end{array}$

Note: 'Clinically significant degression and anxiety' was defined as having a score of at least 10 on either or both of the PHQ-9 and the GAD-7. 'Major depression or GAD' was defined as either having a diagnosis of major depression using the PHQ-9 algorithm, or having a score of at least 5.7 on the GAD-Q-IV, or both.

Abbreviations: GAD: Generaliqged Anxiety Disorder. PHQ-9: 9-item Patient Health Questionnaire Depression Scale; GAD-7: 7-item Generalized Anxiety Disorder Scale; GAD 


\section{Figure 1}

Illustration of prevalence of depression, anxiety and comorbidity.

A: Prevalence rates of clinically significant depression (134/1240 ), clinically significant anxiety ( 186/ 1266 ) and their comorbidity (100/ 1201). Also illustrated are clinically significant depression without anxiety ( 30/ 1245) and clinically significant anxiety without depression ( 77/ 1261).

$B$ : Prevalence rates of current major depression ( 65/ 1240 ), GAD ( 112/ 1269 ) and their comorbidity ( 37/ 1195). Furthermore, current major depression without GAD ( 26/ 1266) and GAD without major depression ( 71/ 1267).

Note: As the number of respondents were different for various measures, different prevalence rates in the figure may not add up to exactly the same figure as that given.

Abbreviations : GAD, Generalized anxiety disorder.
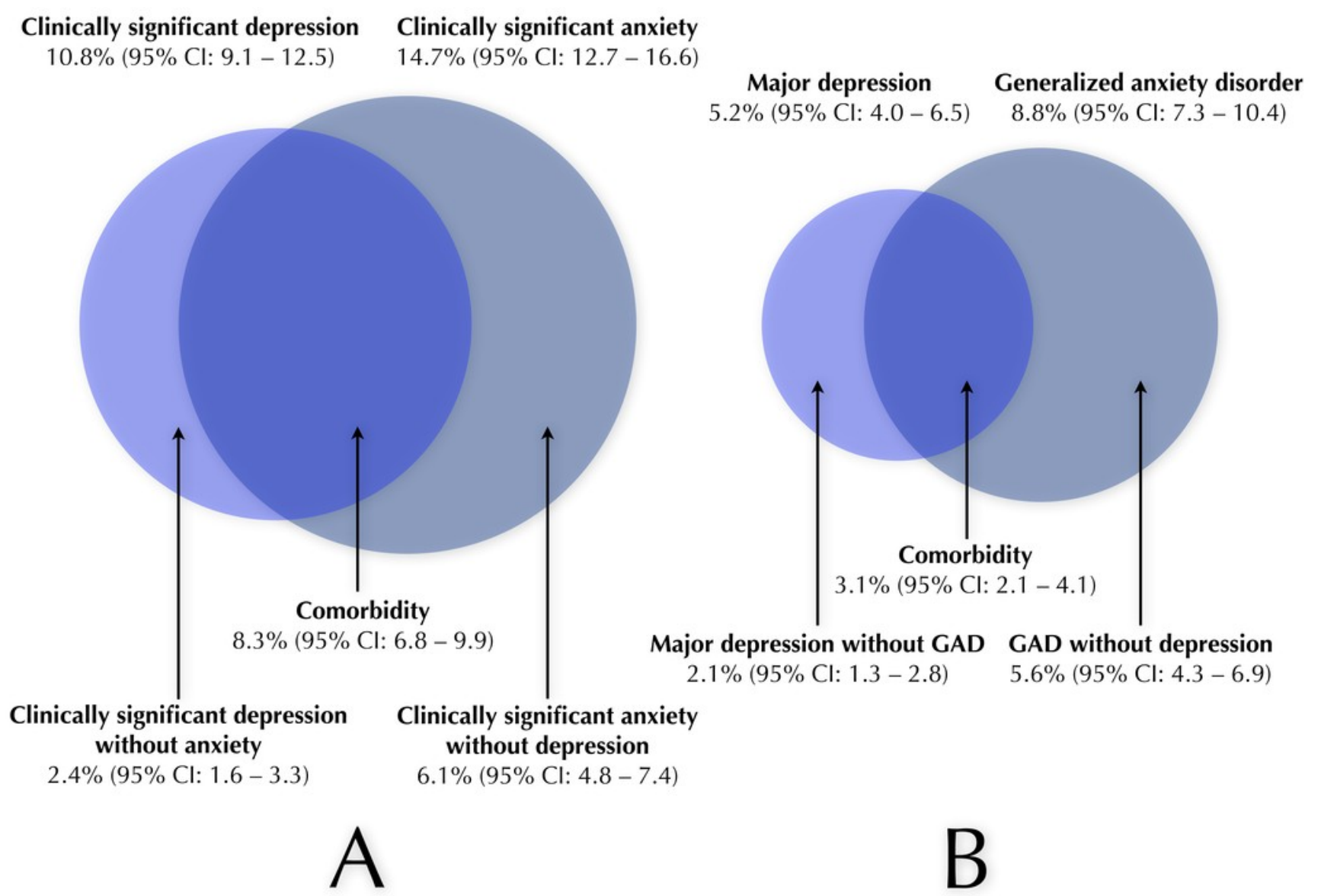

B 